\title{
Special Issue Nutrition and Musculoskeletal Health
}

\author{
Stefania Maggi ${ }^{1}$
}

Published online: 1 June 2019

(c) Springer Nature Switzerland AG 2019
Musculoskeletal conditions include more than 150 conditions globally affecting the locomotor system, and it is estimated that they are the second largest contributor to disability worldwide. Many of these conditions increase with age, such as osteoporosis and associated fragility fractures, osteoarthritis, and sarcopenia. With age, the musculoskeletal system shows increased bone fragility, loss of cartilage resilience, loss of muscular mass and strength, and fat redistribution, leading to a decreased overall physical functioning [1].

Musculoskeletal conditions are, therefore, of great interest in geriatric medicine, since they are responsible for a large percentage of loss of autonomy and reduced ability to participate in social activities, with a major, negative impact on quality of life [2]. The loss of mobility and physical independence resulting from these conditions can be particularly devastating in the older population, also in terms of increased mortality rates $[3,4]$.

In view of the public health relevance that musculoskeletal conditions have in all regions of the world, WHO has proposed recently the Integrated Care for Older People (ICOPE) approach, which identifies the need to improve musculoskeletal function through a range of interventions, with nutrition and exercise as key components [5].

In this special issue of Aging Clinical and Experimental Research, Prof. Jean-Yves Reginster and Dr. Nicola Veronese have collected the most recent clinical and research evidence on the nutritional interventions in the prevention and treatment of musculoskeletal diseases in the aged population. Many outstanding authors have brought to their manuscript a wealth of clinical experience and scientific expertise, and have provided state-of-the art knowledge in this field. I am confident that this special issue will prove to be an invaluable reference for our readers in their research and clinical work.

Stefania Maggi

stefania.maggi@in.cnr.it

1 CNR Aging Branch-NI, Via Giustiniani, 2, 35128 Padua, Italy

\section{Compliance with ethical standards}

Conflict of interest The author states that there is no conflict of interest.

Ethical approval This article does not contain any studies with human participants performed by any of the authors.

Informed consent For this type of study, formal consent is not required.

\section{References}

1. Vos T, Abajobir AA, Abate KH et al (2017) Global, regional, and national incidence, prevalence, and years lived with disability for 328 diseases and injuries for 195 countries, 1990-2016: a systematic analysis for the Global Burden of Disease Study 2016. Lancet 390:1211-1259

2. Beaudart C, Locquet M, Reginster JY et al (2018) Quality of life in sarcopenia measured with the SarQoL ${ }^{\circledR}$ : impact of the use of different diagnosis definitions. Aging Clin Exp Res 30:307-313

3. Filippin LI, Teixeira VN, da Silva MP et al (2015) Sarcopenia: a predictor of mortality and the need for early diagnosis and intervention. Aging Clin Exp Res 27:249-254

4. Sànchez-Riera L, Wilson N (2017) Fragility fractures and their impact on older people. Best Pract Res Clin Rheumatol $31: 169-191$

5. WHO (2017) Integrated care for older people: guidelines on community-level interventions to manage declines in intrinsic capacity. World Health Organization, Geneva (licence: CC BY-NC-SA 3.0 IGO)

Publisher's Note Springer Nature remains neutral with regard to jurisdictional claims in published maps and institutional affiliations. 